\title{
Study on the Performance of SiC Neutron Detector
}

\author{
Mao Li ${ }^{a,{ }^{*}}$, Lifeng $\mathrm{Ji}^{\mathrm{b}}$ and Guofu Liu ${ }^{\mathrm{c}}$ \\ Department of Instrument Science and Technology, National University of Defense Technology, \\ Changsha 410073, China; \\ alimao1993@foxmail.com, b841679571@qq.com, 'Ighlh@163.com
}

\begin{abstract}
Neutron detector performance indexes are the most critical and most direct data which can characterize the detector. In this paper, the mechanism of reaction between $\mathrm{SiC}$ and neutrons is introduced; And the experimental platform for neutron detection is set up. Through the experimental study, the neutron energy spectrum generated by the single energy neutron source is measured by the $\mathrm{SiC}$ detector, and the measurement result is analyzed in depth, so that the detection performance of the detector is evaluated.
\end{abstract}

Keywords: SiC, detector, neutron, radiation.

\section{Foreword}

Because SiC(silicon carbide) semiconductor material has a series of advantages such as stable chemical performance, wide band gap, high breakdown electric field, high carrier saturation drift speed, low dielectric constant, good thermal conductivity and high hardness [1], almost all physical and chemical properties of $\mathrm{SiC}$ is superior to $\mathrm{Si}$. In addition, $\mathrm{SiC}$ is also compatible with $\mathrm{Si}$ integrated circuit technology. Therefore, $\mathrm{SiC}$ is the ideal material of devices with the characteristic of thermal stability, radiation hardening, high voltage, high frequency and high power [2, 3]. Because of these excellent characteristics, the research team developed a kind of neutron detector based on SiC. This paper will study the performance of the detector in detail.

\section{The Mechanism of Reaction between SiC and Neutrons}

When the fast neutrons are incident on the SiC semiconductor radiation detector, the neutron will react with the silicon and carbide atoms in the sensitive region of the detector, and the product of the nuclear reaction is generally charged particles. These charged particles will cause the ionization of the surrounding material, resulting in electron-hole pairs which will be collected by the electrode under the action of the electric field. This collection will generate a signal with the energy information of incident neutrons that can be applied to neutron energy measurement.

The nuclear reaction of SiC and neutrons mainly includes [4]

$$
\begin{aligned}
& \mathrm{n}^{+28} \mathrm{Si} \rightarrow{ }^{28} \mathrm{Si}+\mathrm{n}^{\prime} \\
& \mathrm{n}^{+}{ }^{12} \mathrm{C} \rightarrow{ }^{12} \mathrm{C}+\mathrm{n}^{\prime} \\
& \mathrm{n}^{+28} \mathrm{Si} \rightarrow{ }^{28} \mathrm{Al}+\mathrm{p} \\
& \mathrm{n}^{+{ }^{12}} \mathrm{C} \rightarrow{ }^{12} \mathrm{~B}+\mathrm{p} \\
& \mathrm{n}^{+28} \mathrm{Si} \rightarrow{ }^{25} \mathrm{Mg}+\alpha \\
& \mathrm{n}^{+12} \mathrm{C} \rightarrow{ }^{9} \mathrm{Be}+\alpha
\end{aligned}
$$

Since the silicon and carbon in nature are mainly in the form of ${ }^{28} \mathrm{Si}$ and ${ }^{12} \mathrm{C}$, the most important reaction of the neutron incident detector occurs between neutrons and ${ }^{28} \mathrm{Si}$ and between neutrons and ${ }^{12}$ C ,that is, Eq.(1)-Eq.(6). Eq.(1) and Eq.(2) include neutron elastic scattering and inelastic scattering. Due to the small neutron section and the thin detector depletion layer, the secondary neutrons produced by these two reactions typically penetrate through the detector before causing other reactions. Therefore, the measurement of incident neutron energy can be accomplished by the other four nuclear reactions. 


\section{Experiment Platform}

In order to test the detection performance of $\mathrm{SiC}$ neutron detector, it is necessary to construct experiment system for neutron radiation detection experiment. Due to the limited experimental conditions, single energy neutron sources are not available in this laboratory. All the data acquisition and measurement work of this experiment is completed with the assistance of Institute of Nuclear Physics and Chemistry of China Academy of Engineering Physics in Sichuan Mianyang.

The structure of the experiment platform is shown in Fig.1.

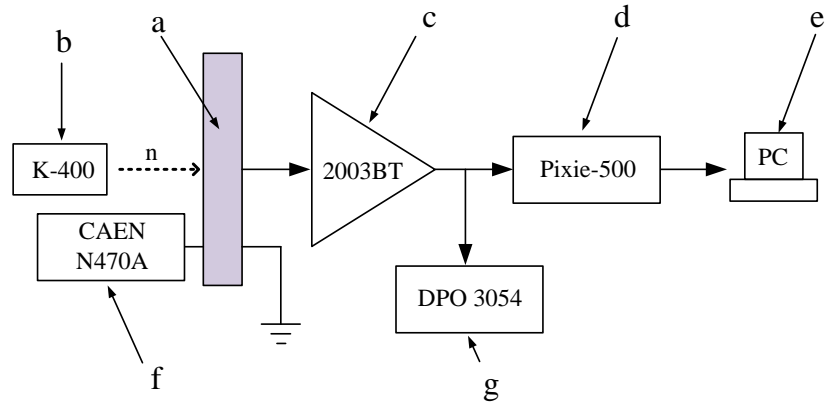

Fig. 1 The structure of the experiment platform

The experiment platform mainly consists of: (a) SiC neutron detectors in different size $\left(25 \mathrm{~mm}^{2}\right.$, $144 \mathrm{~mm}^{2}, 255 \mathrm{~mm}^{2}$ ), (b) accelerated neutron source (K-400), (c) charge sensitive preamplifier (Canberra Model 2003BT), (d) digital spectrometer (Pixie-500), (e) PC, (f) high-voltage power supply (CAEN N470A), and (g) oscilloscope (Tektronix DPO 3054).

The signal generated by the incident neutron and detector is amplified by the charge sensitive preamplifier, and then is transferred into the digital spectrometer when confirming the normal operation of the system by the oscilloscope. The PC is used for neutron energy spectrum acquisition and storage. All the experiments were carried out at room temperature.

\section{Experiment}

\subsection{Neutron Detection Efficiency}

This paper mainly studies the intrinsic detection efficiency of the detector, that is, the ratio of the number $N_{\text {pulse }}$ of pulses to the number of particles incident into the detector. The number of pulses is the sum of the number of particles in all channels. The associated particle method is used to measure the number of particles incident into the detector.

In the experiment, the distance between the $\mathrm{SiC}$ neutron detector and the neutron source is $r=15 \mathrm{~cm}$, and the size of detector is $\mathrm{A}$.A Au-Si surface barrier detector is placed near the neutron source to measure the number $N_{\alpha}$ of associated $\alpha$ particles generated by neutrons source, as shown in Fig.2.

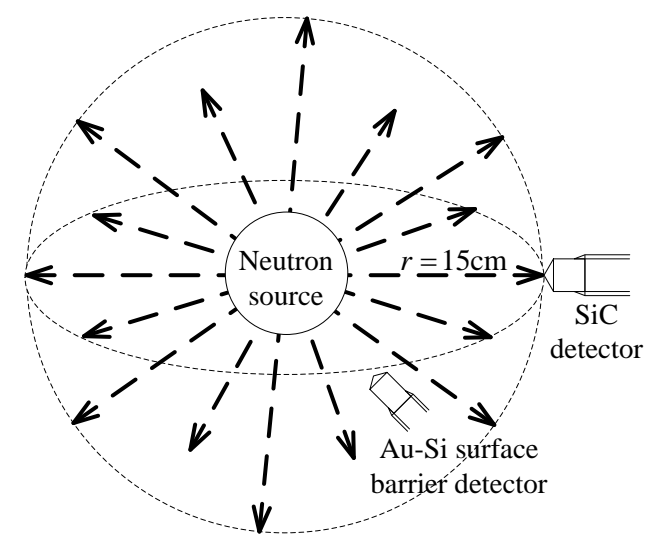

Fig. 2 The position of SiC detector, neutron source and Au-Si surface barrier detector 
According to the relationship between $N_{\alpha}$ and the number $N_{\mathrm{n}}$ of neutrons generated by neutron source

$$
N_{\mathrm{n}}=1.63 \times 10^{7} \cdot N_{\alpha}
$$

the number $N_{\mathrm{n}}$ of generated neutrons in the irradiation time can be calculated. Due to the isotropy of the neutrons generated by neutron source, the relationship between $N_{\mathrm{n}}$ and the number $N_{\mathrm{n}}^{\prime}$ of particles incident into the detector can be expressed as

$$
N_{\mathrm{n}}^{\prime}=N_{\mathrm{n}} \times \frac{A}{4 \pi r^{2}}
$$

which can be used to calculate $N_{\mathrm{n}}^{\prime}$.

In this experiment, $N_{\text {pulse }}$ detected by the detectors in different size and $N_{\alpha}$ were obtained with the irradiation time of 20 minutes. The neutron detection efficiency can be obtained, as shown in table 1 .

Table 1 The detection efficiency of the detectors in different size

\begin{tabular}{|c|c|c|c|}
\hline Detector Size & $25 \mathrm{~mm}^{2}$ & $144 \mathrm{~mm}^{2}$ & $225 \mathrm{~mm}^{2}$ \\
\hline$N_{\alpha}$ & 3878398 & 3790050 & 3739199 \\
\hline$N_{\text {pulse }}$ & 285157 & 1676157 & 2708261 \\
\hline Detection efficiency $\left(10^{-5}\right)$ & 5.1015 & 5.3274 & 5.5839 \\
\hline
\end{tabular}

The neutron detection efficiency of the detector in the size of $25 \mathrm{~mm}^{2}$ under different bias was detected, as shown in table 2.

Table 2 The detection efficiency of the detectors under different bias

\begin{tabular}{|c|c|c|c|c|}
\hline Bias & $25 \mathrm{~V}$ & $35 \mathrm{~V}$ & $45 \mathrm{~V}$ & $55 \mathrm{~V}$ \\
\hline$N_{\alpha}$ & 3282716 & 3884891 & 3878398 & 3896714 \\
\hline$N_{\text {pulse }}$ & 222273 & 286941 & 302568 & 316446 \\
\hline Detection efficiency $\left(10^{-5}\right)$ & 4.6981 & 5.1248 & 5.4130 & 5.6346 \\
\hline
\end{tabular}

The results show that the detection efficiency of the detector can be improved by increasing the size of the detector and increasing the bias. However, due to the small reaction cross-section between $\mathrm{SiC}$ and neutrons, most of the neutrons and SiC material cannot react effectively and penetrate through the detector directly. In addition, the energy of the $\alpha$ particles and protons produced by the reaction between neutrons and $\mathrm{SiC}$ material cannot be completely absorbed in the detector, which result that the amplitude of part of pulses is less than threshold set in acquisition and most of the neutrons cannot be recorded. Therefore, most of the neutrons cannot be recorded, which result in the lower detection efficiency.

\subsection{The Performance of Neutron Spectrum Measurement}

The pulse amplitude spectrum of the neutrons with the energy of $14 \mathrm{MeV}$ is measured by different size of detectors as shown in Fig.3.

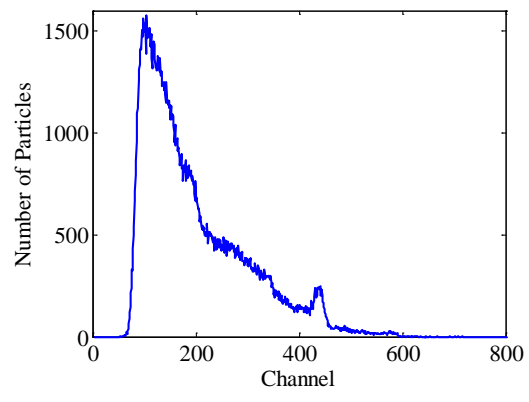

(a)

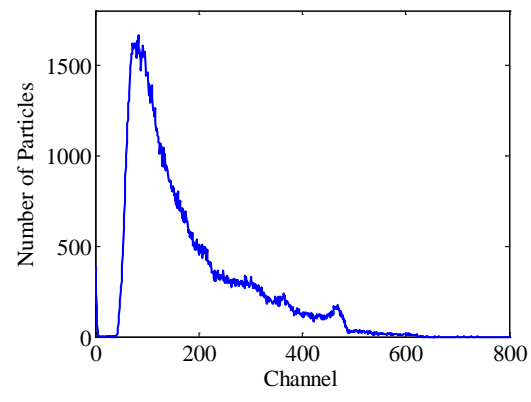

(b)

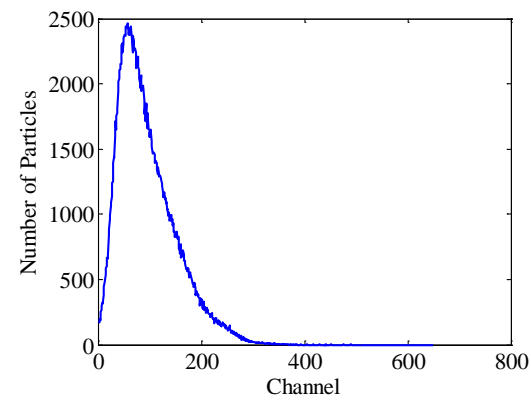

(c)

Fig. 3 The pulse amplitude spectrum measured by different size of detectors (a) $25 \mathrm{~mm}^{2}$; (b) $144 \mathrm{~mm}^{2}$; (c) $225 \mathrm{~mm}^{2}$

The pulse amplitude spectrum is converted to neutron spectrum, as shown in Fig.4. In the spectrum of $25 \mathrm{~mm}^{2}$ and $144 \mathrm{~mm}^{2}$, a significant peak can be seen the energy of $8.7 \mathrm{MeV}$, which is formed by the superposition of the energy peaks of ${ }^{28} \mathrm{Si}(\mathrm{n}, \alpha){ }^{25} \mathrm{Mg}$ and ${ }^{28} \mathrm{Si}(\mathrm{n}, \mathrm{p}){ }^{28} \mathrm{Al}$; In the range of 
$6 \sim 8 \mathrm{MeV}$ and $9 \sim 11 \mathrm{MeV}$, the energy of ${ }^{28} \mathrm{Si}(\mathrm{n}, \alpha){ }^{25} \mathrm{Mg},{ }^{28} \mathrm{Si}(\mathrm{n}, \mathrm{p}){ }^{28} \mathrm{Al}$ and ${ }^{12} \mathrm{C}(\mathrm{n}, \mathrm{p}){ }^{9} \mathrm{Be}$ is close to each other, which result in a continuous spectrum, and there is no significant peak. When $14 \mathrm{MeV}$ neutrons collide with $\mathrm{SiC}, \mathrm{Si}$ and $\mathrm{C}$ atoms carry the energy of $3.98 \mathrm{MeV}$ and $1.862 \mathrm{MeV}$ respectively, and the corresponding energy peaks should exist theoretically. However, because of the random position of reaction, most of the neutrons react near the edge of the sensitive region. Therefore, a large number of particle events are recorded at $0 \sim 6 \mathrm{MeV}$, and the two theoretical peaks are flooded in other events, with no significant peaks in the spectrum.

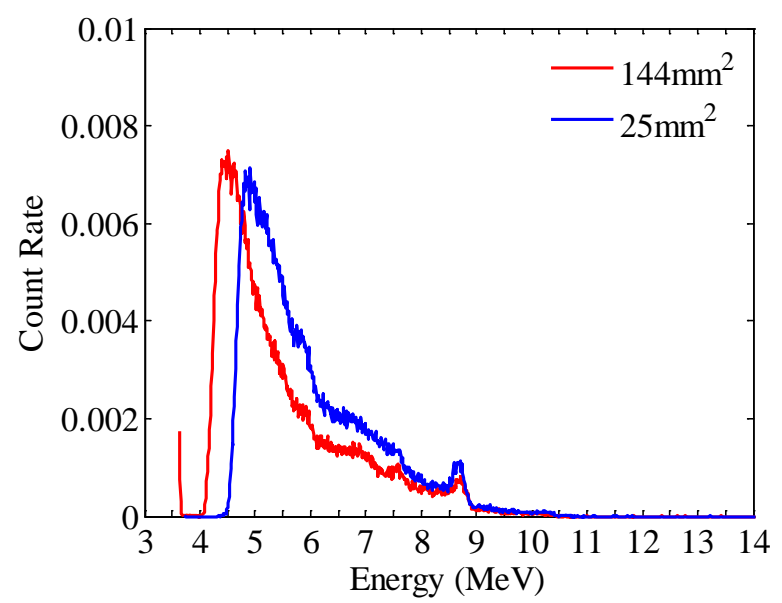

Fig. 4 The neutron spectrum of $25 \mathrm{~mm}^{2}$ and $144 \mathrm{~mm}^{2}$

As the capacitance of detector increases with the increase of the detector size, the noise caused by capacitance become stronger significantly. Thus there is no significant peak in the spectrum of $225 \mathrm{~mm}^{2}$. And the detection efficiency of the detector increases insignificantly with the increase of the detector size. Therefore, it can be considered that as the size of the detector increases, the detection performance of the detector deteriorates drastically. And the size of detector should be diminished moderately to get the better performance of the detector.

The energy spectrum of $25 \mathrm{~mm}^{2}$ at $8.66 \mathrm{MeV}$ was fitted with Gaussian fitting. The results are shown in Fig.5. The energy resolution can be expressed as the ratio of FWHM(full width at half maximum) to the energy of the peak. The energy resolution of SiC detector can be calculated as 3.9\%, which is of good performance.

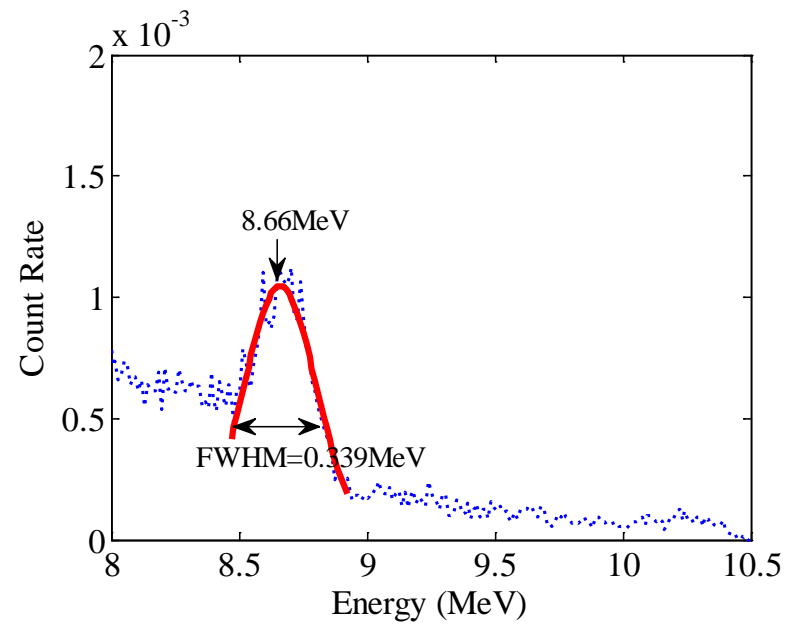

Fig. 5 The Gaussian fitting of spectrum at $8.66 \mathrm{MeV}\left(25 \mathrm{~mm}^{2}\right)$

In addition, the spectral performance of the detector was tested under different bias voltages. It can be seen from Fig. 6 that the energy scale under different bias is in good agreement. Within a certain range of bias, the energy spectrum peak gradually becomes sharper with the increase of the bias voltage, that is, the energy resolution of the detector becomes better. 


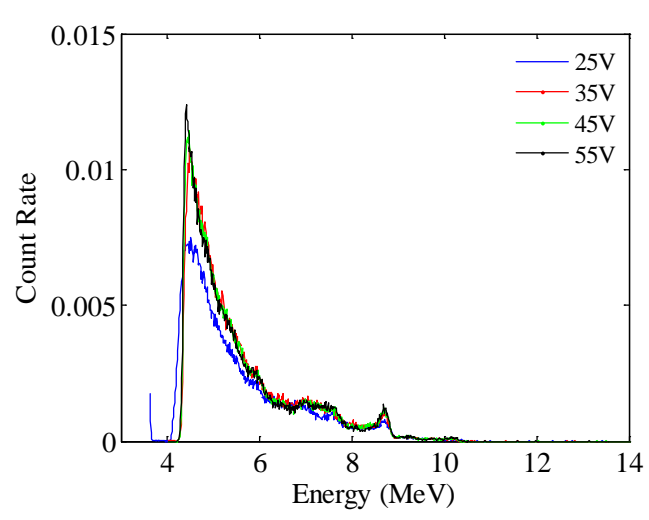

(a)

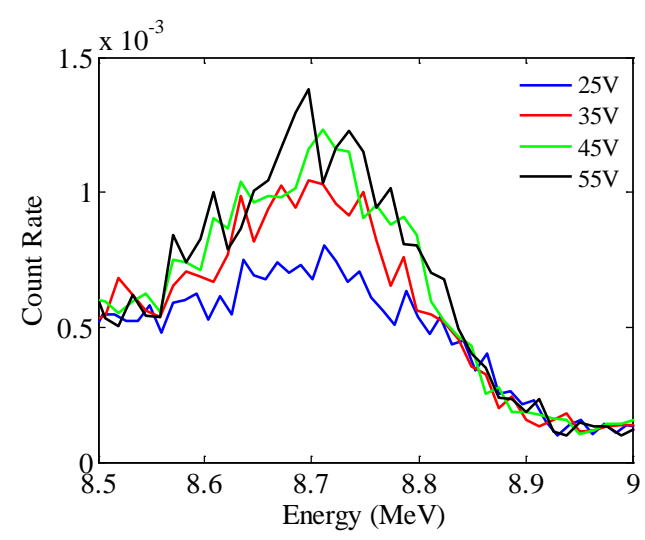

(b)

Fig. 6 The neutron spectrum under different bias $\left(25 \mathrm{~mm}^{2}\right)$

(a) The whole spectrum; (b) The spectrum at $8.66 \mathrm{MeV}$

\section{Summary}

In this paper, the detection performance of previously developed $\mathrm{SiC}$ neutron detection is studied. The experimental platform for measuring the neutron energy spectrum of the detector is built. The neutron detection performance was tested by using a $14 \mathrm{MeV}$ single energy neutron source. The experimental results show that the silicon carbide neutron detector has good detection performance and the energy resolution is $3.9 \%$. And it is found that reducing the size of the detector and improving the reverse bias can improve the detection performance.

\section{Acknowledgements}

This project was financially supported by the National Natural Science Foundation of China under Grant no. 11375264.

\section{References}

[1] JIN Gen, CHEN Fa-guo, YANG Ya-peng, XU Yuan and WANG Xi-tao, SiC Semiconductor Detector with Temperature and Radiation Hardness, J. Nuclear Electronics \& Detection Technology, 30 (2010) 909-912.

[2] Runkle R C, Bernstein A, Vanier P E, Securing Special Nuclear Material: Recent Advances in Neutron Detection and Their Role in Nonproliferation, J. Journal of Applied Physics, 108 (2010) 111101.

[3] Wright N G, Horsfall A B, SiC sensors: a review, J. Journal of Physics D Applied Physics, 40 (2007) 6345.

[4] F. Fasolo, F.H. Ruddy, Silicon Carbide Neutron Detectors, J. InTech, Rijeka Croatia, 28 (2011) 276-293. 